\title{
Quantitative Comparison of Partial-Full Fingerprints at Scenes Using a Hand-Held Digital Device: A Quasi-Experimental Design Study
}

\section{Forensic}

\section{Investigation}

\author{
John Zheng Wang* \\ School of Criminology, Criminal Justice and Emergency Management, \\ California State University, Long Beach, USA
}

\section{*Address for Correspondence}

John Zheng Wang, Forensic Studies Program, School of Criminology, Criminal Justice and Emergency Management, California State University, Long Beach, CA 90840, USA, Tel: 562-985-4741; E-mail: zwang2@csulb.edu

Keywords: Forensic science; Crime scene investigation; Criminalistics; Partial fingerprint; Partial-full print examination; Quantitative measurements; Quasi-experimental design; Portable digital devices at crime scenes

\begin{abstract}
A partial-full fingerprint comparison continues to be one of the main challenges faced by fingerprint examiners in the lab. This challenge becomes even greater when such a partial-full print comparison is needed for a preliminary decision of an exclusion decision at the crime scene by investigators. This study intends to employ a hand-held digital device to perform quantitative measurements for partial-full fingerprint examination. Based on a quasi-experimental design to simulate partialfull fingerprint situations at crime scenes, this study was able to measure $(\mathrm{mm})$ the samples quantitatively in a real time manner. The results of the digital comparison between partial prints $(<35 \%)$ and full prints $(>95 \%)$ indicate that a decision of a match (the same source) or not a match can be achieved on three digital geometrical modes: liner and angular measurements, and a matrix combination of both. The technical results of this study suggest three important implications: 1) to provide a portable device with quantitative measurement functions in the field; 2) to help increase the success rate of IAFIS in the police station; and 3) to recommend an operational protocol for a partialfull fingerprint comparison. The practical implications of initial exclusion and later inclusion from this study may present a possible methodology to address the challenges more effectively.
\end{abstract}

\section{Introduction}

Matching a partial or incomplete fingerprint to a full or complete fingerprint still remains a challenge in real world applications because partial fingerprints developed and lifted from crime scenes are much smaller, more broken, and less clear. Currently, partial fingerprints tend to have relatively low success rates for meeting IAFIS examination standards due to the limitations inherent in its algorithm. When partial fingerprints are unused, cases often become stagnant or "cold" and many valuable opportunities for solving crimes are lost. In reality, partial fingerprints constitute nearly eighty percent of prints found at crime scenes, including many terror-related incidents [1].

While the palm-sized device used for this study was part of the author's research involvement in another project, a similar device can be purchased online under "digital scopes," namely "AmScope". The digital scope is connected to a laptop via a USB, thus being portable for a crime scene examination. The device is able to take digital images (JPEG) of partial and full fingerprints for comparison, which makes it easier for online communication and evidence storage. If necessary, the device is capable of connecting to a projector for a live analysis and comparison at any location, such as crime scenes, police
Submission: 01 July, 2016

Accepted: 21 July, 2016

Published: 25 July, 2016

Copyright: @ 2016 Wang JZ. This is an open access article distributed under the Creative Commons Attribution License, which permits unrestricted use, distribution, and reproduction in any medium, provided the original work is properly cited.

Reviewed \& Approved by: Dr. Robert Allen, School of Forensic Sciences, Oklahoma State University, USA

departments, labs, DA's offices or even in courtrooms for an expert's testimony. Further, the device can be used with black/white, UV, infrared, and polarized light sources, each with a magnification of up to 200X. Most importantly, the new device can provide micro-digital measurements, which is a very practical comparison technique for a partial-full fingerprint examination at scenes. The measurement unit can be selected at $\mathrm{mm}$ or inch $(0.001)$.

Although the use of fingerprints as an official means of identification and verification by police has occurred for just more than the past 100 years, the current IAFIS is capable of image scanning, optical storage, image compression, and quick processing power $[2,3]$. Recently, the IAFIS incorporates biometric components such as ABIS (Automated Biographic Information System) otherwise known as a NGIS (Next Generation Identification System) [4]. These technologies are systems because they use computers, software, and live-scans and can interact with other subsystems at the local, regional, national, and international levels so long as participating agencies share the IAFIS systems [5].

\section{IAFIS and live-scan technical requirements}

According to "AFIS Requirements Specification Version 1 [6]", the following are three major highlights of the IAFIS's technical components. First, the system can perform a latent-to-ten-print identification search where an automatic $1: N$ search occurs between a latent print against all ten print records in the on-line fingerprint record database. Second, the system can run a latent-to-unsolved latent fingerprint identification search where an automatic 1: $\mathrm{N}$ search occurs between a latent fingerprint against all finger records in the database. Third, the system requires the submitted record of the image meet minimum image quality standards for automatic input

Endnote: The preliminary results were presented at the $66^{\text {th }}$ Annual Conference of the American Academy of Forensic Sciences, February 17-22, 2014, in Seattle, WA. 
processing [7].

As operational requirements, both IAFIS and live-scan require a high quality of latent fingerprint image for submission. Furthermore, one of the leading fingerprint articles suggests a minimum 10-12 matched minutiae are required for a matching decision [8]. Otherwise, any sub-standard or poor quality fingerprint image may result in less accurate candidates automatically identified by the system. In terms of the live-scan mode of input, certain specific requirements are set: 1) the image must be captured at a spatial resolution of 1,000 pixels per inch (ppi), with 8-bit (256 level) gray scale quantization; 2) the captured image must be a full rolled (tip to crease or nail to nail) fingerprint image; and 3) if possible, the latent type (finger or palm, if known), finger position (if known), and/or linkages to other latent images in a multi-finger lift should be specified in order to reduce the search time [9]. In reality, many partial fingerprints (with less than $35 \%$ area) simply do not possess such qualities for submission and comparison.

\section{The report from the NRC}

In 2009, the National Research Council published Strengthening Forensic Science in the U.S.: A Path Forward. This landmark report challenged many current evidentiary examinations in the field, in the lab, and in the courtroom as less scientific because traditional forensic examinations of evidence rely upon an examiner's subjective judgments of the pattern and the levels of minutiae [10]. The decision of exclusions/inclusions usually lacks scientific and objective quantifiable measurements found in other forensic methods such as DNA analysis. The report strongly recommends forensic examination of pattern evidence contain quantifiable measurements, reliable methodology, and scientific procedure based on scientific research studies. In essence, the challenge lies in seeking more novel techniques, which allow statistical or quantitative analyses [11].

\section{New attempts}

Due to the dramatic increase in the database sizes and increased attention by the courts to the adequacy of proof for latent print positive identifications, the IAFIS measures each fingerprint image submission and rejects those of sub-standard quality. While there have been several reports on partial fingerprint examination, these studies still suffer from three practical implications: lack of practical methods, lack of portable devices, and lack of field protocols [12-14]. The three are key factors for crime scene technicians or investigators to perform a real time partial-full print comparison at the scene. Therefore, there exists a need to develop partial-full print examination methods using portable devices with quantitative measurements by practical protocols. Certainly, an examination of partial-full prints with probative values at the crime scene would improve the efficiency of crime scene investigation.

\section{Materials and Methods}

Current fingerprint software and IAFIS database employ digital technology for a comparison via probability analysis or candidate-scoring thresholds. However, IAFIS relies solely on the algorithm recognition of pattern and minutia characteristics (ridge ending, bifurcation, and orientation of both) between the known and the unknown samples without any statistical and geometrical measurements for a match or a non-match decision, thus considered as less scientific by some critics [10].

\section{Operational definition of partial fingerprints}

As an operational definition of a partial fingerprint, this study uses the following criteria for sampling selection:

1. A partial fingerprint is a print with much smaller usable or legible area of pattern or minutia features than a full fingerprint. The unusable or illegible portions vary depending on the condition and quality of the partial fingerprint deposited, developed and lifted;

2. A partial print usually has a partial core or delta, on which many minutia features exist;

3. This study selects partial print samples with approximate $35 \%$ area with pattern and minutia features due to the minimum requirement of statistical discrimination power.

While many partial fingerprints also contain other noisy components, such as distorted, overlapped, smeared, and/or smudged portion of pattern and minutia features, this study intends to focus only on a partial fingerprint with around 35\% area with usable and legible features. This study also contends an inclusion or identification of two fingerprints (known vs. unknown) be based on both quality and quantity levels in terms of sufficient pattern and minutia features that two prints should come from the same source. On the other hand, a decision of an exclusion of the two fingerprints should also rely upon the sufficient pattern and minutia features at both quality and quantity levels should the two prints not come from the same source. The qualitative and quantitative approaches should better address a situation where a partial fingerprint is collected and no algorithm with the capacity is available to provide calculated quantity scores to assist the operator in making, editing, accepting, or rejecting a decision [15].

\section{Quasi-experimental design}

In order to reflect a partial-full fingerprint situation at a crime scene, this study selected samples based upon a quasi-experimental design. A quasi-experimental design is an empirical method estimating the causal impact of a treatment or an intervention on its targeted samples. The design consists of three main components. First, quasi-experimental research does not use random assignments to treatment or control. Instead, a quasi-experimental design typically allows the researcher to control the assignments to the treatment condition and use some criterion for practical purposes. Second, the researcher can have control or manipulation over assignments to the treatment due to limitations of time and resources. Finally, there are several types of quasi-experimental designs, each with different strengths, weaknesses, and applications [16]. This research study and data collection follows a quasi-experimental design with three subtype components (pretest versus posttest, instrumental functions, and propensity score matching) in steps, making the collection and comparison of the partial-full fingerprints possibly similar to real crime scene situations in the field.

\section{Sampling}

Sample fingerprints $(n=1,000)$ from 12 different surfaces 
(commonly seen in a household) were collected as a purposive sampling from the researcher's mock crime scene training sessions over the past five years, which qualified as a quasi-experimental setting and was quite similar to real crime scene conditions. Pairs of trainees lifted the fingerprints deposited using regular magnetic powder, which allowed for sets of possible identical fingerprints of partial and full prints by each pair of trainees. Each of the 100 bags contained ten prints deposited, developed, and lifted by two trainees. The ten prints in each bag were screened out first into two categories by percentage of area of legibility: $>95 \%$ and $<50 \%$. For a cluster sampling to reduce the threat of a lack of internal validity, the samples in 100 bags with two categories were further examined and classified further into four categories by percentages of legibility: $>95 \%$; $<75 \%$; $<50 \%$, and $<35 \%$. As manipulation attempts, the quasi-experimental design renders the treatment samples to be proximately comparable at a baseline. Under a criteria sampling, only bags containing fingerprints with $>95 \%$ legibility (resembling the fingerprints from the suspect's inked fingerprints or in the database) and partial fingerprints with < $35 \%$ legibility (as unknown prints imitating latent prints lifted from crime scenes) in a similar pattern were finally selected for 50 pairs.

\section{Pattern selections}

Each pair now contains a $<35 \%$ partial print and a $>95 \%$ full print and they may or may not have come from the same source from the two trainees. The current statistics indicate that the loop pattern approximately accounts for $60 \%$, the whorl pattern for $35 \%$, and the arch pattern for $5 \%$ in the natural distribution. Due to time limitations of the research, from the 50 pairs qualifying the selection standard, the final twenty pairs (> 95\% versus $<35 \%$ ) were chosen to proportionally reflect the three $60 \%, 35 \%$ and $5 \%$ distributions: twelve pairs of the loop pattern, six pairs of the whorl pattern, and two pairs of the arch pattern.

\section{ACE-V protocol}

The researcher then used the hand-held digital device to perform the analysis, comparison, and evaluation of the final twenty pairs as model samples (the treatment). Both regular magnifiers (3X) and stereo microscopes (10X) were used with the ACE steps and then verified by a colleague for the pattern selection. Three digital measurement modes (linear, angular, matrix measurements) were performed to compare each pair of the partial-full fingerprints. Although the device is able to provide ten geometric measurements, desirable comparison results were all obtained after applying the three geometric formats. Finally, the paired images between the pre- and the post-test were photographed (JPEG) and reported in six figures for a demonstration purpose. All the comparisons of the sample partial-full prints were performed in real time examination settings and the results were verified again by the colleague.

\section{Results}

After selecting the prints for comparison, six images from three pairs in three different patterns (loop, whorl, and arch) were provided for a demonstration with each pair containing one pre-test and one post-test image. In order to secure maximum internal validity and minimum error rates, the magnification for the six comparison images in three pairs was all set at $25 \mathrm{X}$ and with the same working distance so the need for calibration is minimally required.

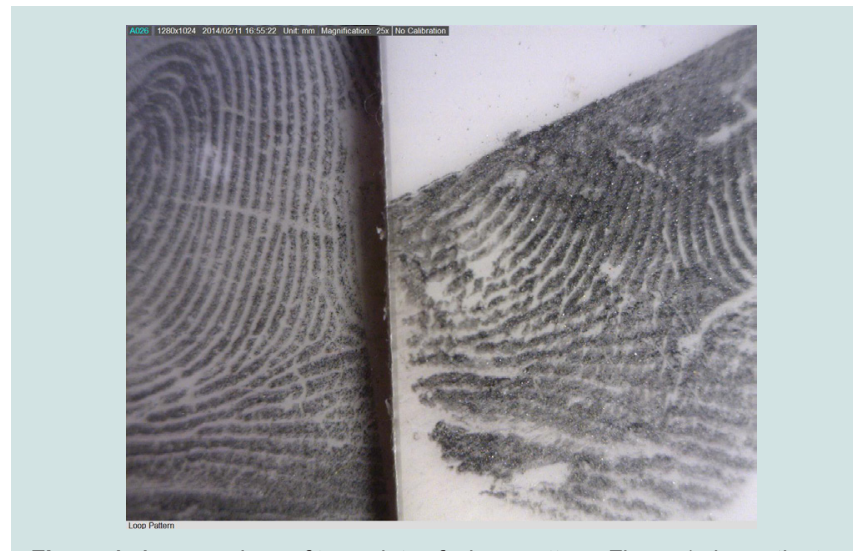

Figure 1: A comparison of two prints of a loop pattern. Figure 1 shows the two pre-test images produced with the device.

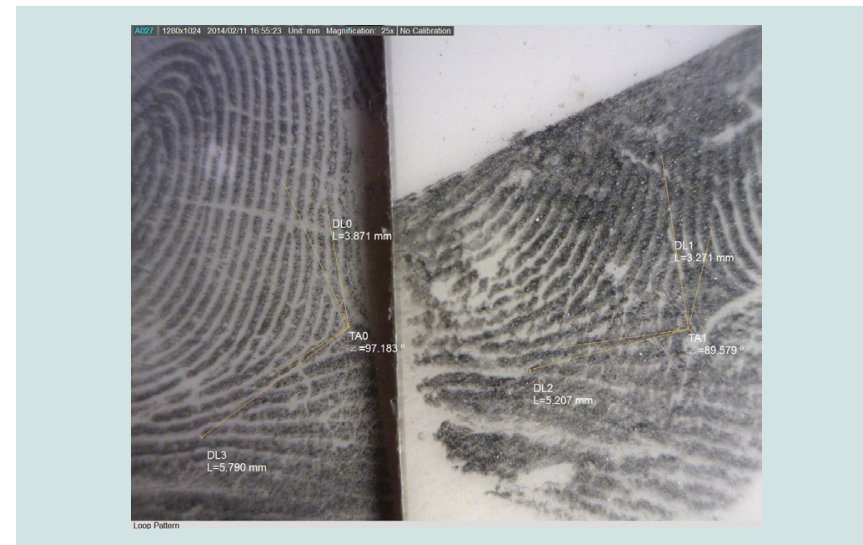

Figure 2: A comparison of two prints of a loop pattern. Figure 2 presents the two post-test images produced with the device.

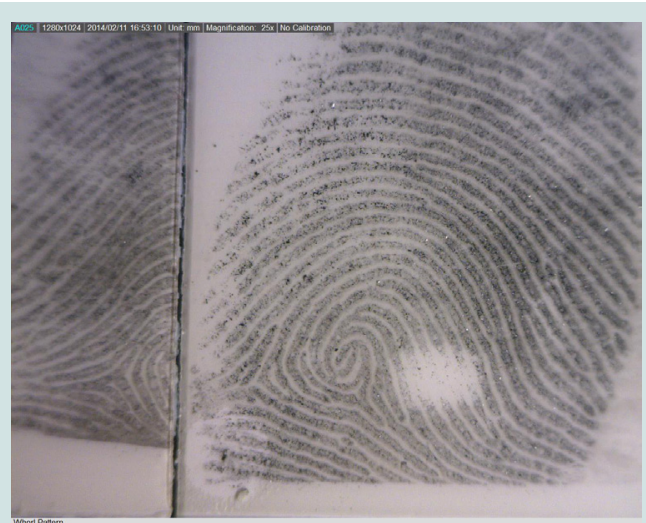

Figure 3: A comparison of two Prints of a whorl pattern. Figure 3 illustrates two pre-test images produced with the device.

Figure 1 presents a pair of a partial print and a full print of a loop pattern. The partial print has approximately $30 \%$ area of a full print with a partial delta, which is very similar to the next full print. The partial print can hardly produce any comparison result by the IAFIS standard, thus making it one of the samples for the quasiexperimental design study. Figure 2 provides two post-test images after the treatment (the processing by the hand-held device). From the digital quantification, the researcher obtains three quantifiable 
Citation: Wang JZ. Quantitative Comparison of Partial-Full Fingerprints at Scenes Using a Hand-Held Digital Device: A Quasi-Experimental Design Study. J Forensic Investigation. 2016; 4(2): 6.

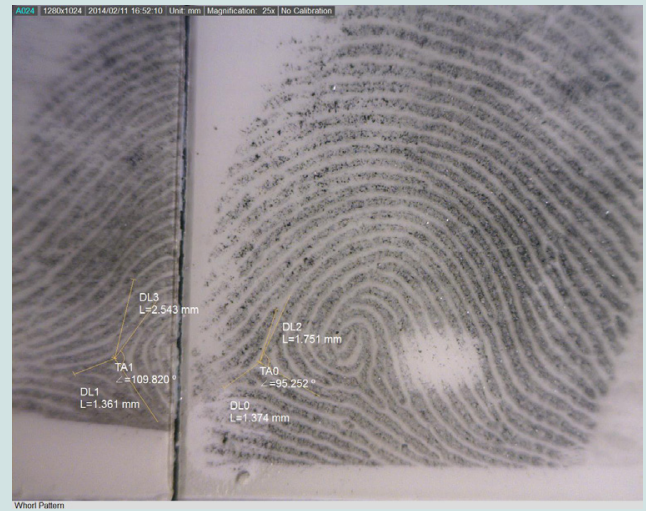

Figure 4: A comparison of two prints of a whorl pattern. Figure 4 gives the two post-test images produced with the device.

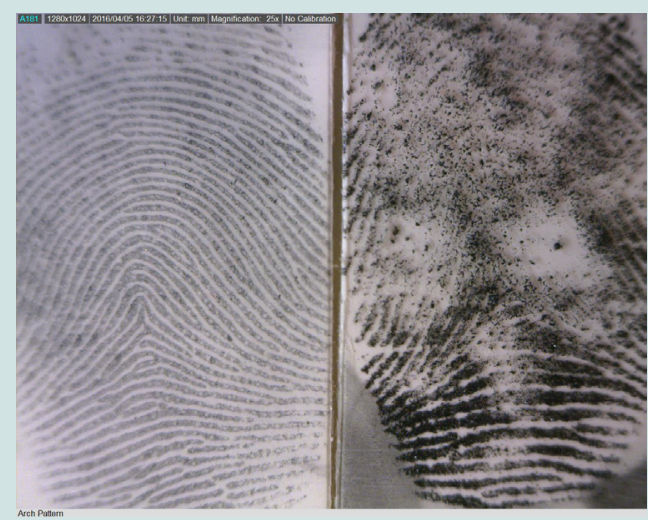

Figure 5: A comparison of two prints of an arch pattern. Figure 5 demonstrates the two pre-test images produced with the device.

measurements. First, DL0 (from the right tip of the delta to the joint point of the bifurcation) in the full print is $3.871 \mathrm{~mm}$ in distance with a left slant, while a similar distance (DL1) in the partial print is 3.271 $\mathrm{mm}$, but the direction is right slanted. Second, DL3 (from the right tip of the delta to the lower point of a ridge ending) in the full print is $5.709 \mathrm{~mm}$. A similar position distance in the partial print is $5.207 \mathrm{~mm}$ with a different level of slope. Third, the key difference between the two prints lies in the two angles (TA0 and TA1) of the delta boundaries ( $97.183^{\circ}$ in the full versus $87.579^{\circ}$ in the partial). Accordingly, based on both quality and quantity levels, the partial print and the full print are determined to be not from the same source.

Figure 3 illustrates two pre-test prints of a whorl pattern. The partial print has a similar portion of details as the full print. The right lower portion has approximately $30 \%$ area of a full print with a partial delta. Again, the partial print can hardly produce any comparison result by the IAFIS standard, thus making it one of the samples for the quasi-experimental design study.

Figure 4 presents two post-test images after the treatment (the processing by the hand-held device). The digital quantification is able to produce three quantifiable measurements. First, DL0 (from the left tip of the delta to the nearest joint point of the bifurcation toward the left lower direction) in the full print is $1.374 \mathrm{~mm}$ in distance while a similar distance in the partial print is $1.361 \mathrm{~mm}$ (DL1) with a different level of slope. Second, DL2 (from the left tip of the delta to the nearest ridge ending toward the left upper direction) in the full print is 1.751 $\mathrm{mm}$. However, there is no corresponding ridge ending in a similar direction in the partial print, rather a joint point of a bifurcation with $2.543 \mathrm{~mm}$ in distance (DL3). Third, the key difference between the two prints is the two angles (TA0 and TA1) of the two delta boundaries in both prints $\left(95.252^{\circ}\right.$ in the full versus $109.820^{\circ}$ in the partial). Thus, based on both quality and quantity levels, the partial print and the full print are determined to be not from the same source.

Figure 5 gives two pre-test prints of an arch pattern. The partial print has a roughly similar contour as the full print. The details of legibility are of approximately $20 \%$ area of a full print in both lower portions.

Figure 6 presents two post-test images after the treatment (the processing by the hand-held device). From the digital quantification, three quantifiable measurements can be obtained: 1) DL0 (from the first ridge ending below the baseline of the arch to the nearest joint point of a bifurcation below) in the full print is $1.825 \mathrm{~mm}$ (DL0) in distance while a similar position distance in the partial print is 2.051 $\mathrm{mm}$ (DL1). However, the direction of the bifurcation in DL0 is pointed to the right while the direction of DL1 is toward to the left; 2) DL2 (from the ridge ending to another ridge ending of a bifurcation) in the full print is only $3.395 \mathrm{~mm}$. A similar position distance in partial print is $5.008 \mathrm{~mm}$; 3 ) the key difference between the two prints lies in the two angles (TA0 and TA1) of the two lines in both prints $\left(39.471^{\circ}\right.$ in the full versus $61.42^{\circ}$ in the partial). Therefore, based on

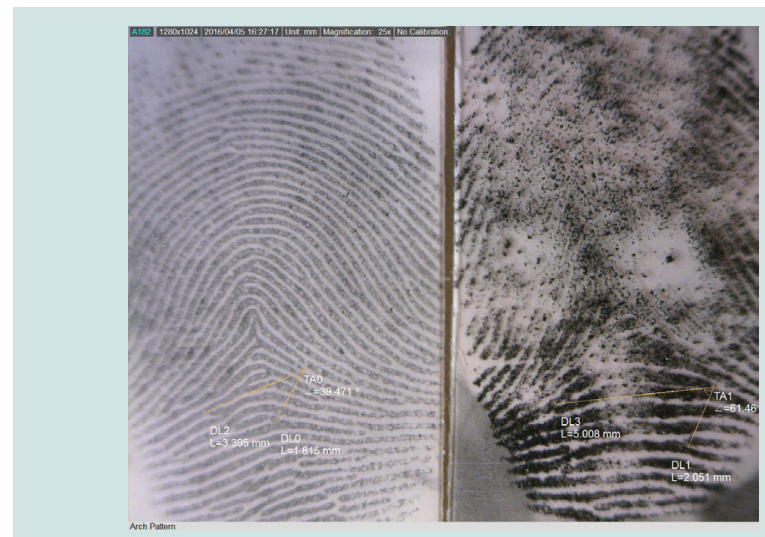

Figure 6: A comparison of two prints of an arch pattern. Figure 6 provides the two post-test images produced with the device.

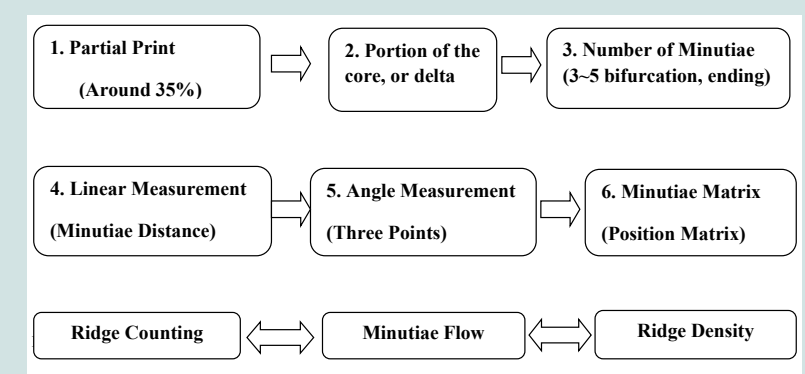

Figure 7: Systematic flow of quantification method (Operational protocol). Figure 7 proposes a protocol of partial-full prints comparison based on the quasi-experimental study. 
both quality and quantity levels the partial print and the full print are determined to be not from the same source.

\section{Protocol for Partial-Full Fingerprint Comparison at a Crime Scene}

From the quasi-experimental study, a protocol is proposed for partial-full print comparisons in six steps for a crime scene investigation purpose:

1) Determine what size in percentage (\%) of the partial fingerprint is legible. Because this study is the first phase of a continuous project, the researcher selected partial fingerprints with good legibility around 35\% as research targets.

2) Determine whether the partial fingerprint contains a core or delta area(s)--a preferable base point to start with.

3) Determine how many minutiae both prints contain under a magnifier, preferably from three to five minutiae in the same location.

4) Locate the two minutiae on the two images (the partial and the full), and measure the distance between them using the linear measurement.

5) Locate three minutiae in both the partial and the full prints, and measure the angle among the three points.

6) Compare the linear distance in millimeters, the angle among the three points, and the matrixes between the two images in terms of minutiae positions.

Figures 1-6 presented the examination process of the analysis, comparison, evaluation, and verification via digital and geometrical measurements, and summarize the systematic flow of the quantification method. If a supplemental reference is needed, ridge counting, minutiae flow, and ridge density may be used.

\section{Discussion}

The NAS report of 2009 criticizes many pattern examinations to be a more subjective nature. The forensic science community thus has come to a crossroad whereas both qualitative and quantitative measurements are critical in courtroom battles. This study intends to provide a new direction for partial-full fingerprint examinations and has great potential for application to other types of evidentiary examinations such as for casings, bullets, and hair to provide more reliable and valid methodologies in the future.

This quasi-experimental project provides a novel approach and offers four levels of forensic significance. First, a special hand-held device is able to examine partial-full prints in the field. The new portable device is a palm-sized tool connecting to a laptop and allows for a quick comparison on-site by crime scene investigators. This technology can save crime scene technicians as well as lab examiners valuable time and resources when compared to the standard practice of treating partial-full fingerprints. Second, three geometrical measurements (linear, angular and matrix) are employed for a quick comparison at scenes.

This novel method is able to provide quantifiable statistics in real time via geometrical measurements between minutiae that are on both unknown and known fingerprints for an exclusion or inclusion purpose. This method and technology answers the challenges from the National Research Council's 2009 report Strengthening Forensic Science in the U.S.: A Path Forward. The report challenged many current evidentiary examinations in the field, in the lab, and in the courtroom as less scientific because many forensic examinations rely upon an examiner's visual perception of pattern and minutiae features and lack quantifiable measurements found in other forensic methods such as DNA analysis.

Third, an operational protocol is proposed and tested for comparison allowing an examination result to rely upon the number of minutiae, the minutiae position, and digital geometrical measurements. Therefore, this new method also qualifies the entire fingerprint examination process of ACE-V--analysis, comparison, evaluation, and verification. The new device has much wider applications for other criminal justice related settings such as in the lab, the DA's office, trial demonstrations, and even in a remote long-distance communication via $\mathrm{Wi}$-Fi/Internet due to its portable, digital and quantifiable features. Finally, the comparison results are straight forward and are capable of comprehension by laypersons (e.g. jurors) since the basis relies upon common linear, angular, and matrix measurements in millimeters, thus realizing the adage of "a picture is worth of a thousand words." With the three unique features of being portable, digital, and quantifiable, this new device is a possible tool in both preliminary examinations of partial-full prints at crime scenes as well as performing a supplemental or verification examination in the lab.

\section{Future implications}

The partial-full print comparison has certain practical implications for crime scene investigation by making partial fingerprints useful for otherwise unsolvable cases. The researcher suggests several directions. Manufacturers should offer simplified "forensic package" software equipped with only the three most important geometrical measurements (linear, angular, and matrix) for an easy use. Since this partial-full print comparison is a non-destructive method, examiners should field-test the device for greater validity and reliability. For the same reason, post-conviction verifications should employ this new device for partial-full fingerprint evidence. Finally, the new digital viewer is an important approach to developing objective criteria for the analysis of pattern evidence. More than just the approach, this methodology is portable and thus enables useful on-scene examination for preliminary analysis of fingerprint evidence. For the next step, a mixed method with positive and negative control tests may certainly reduce the limitations of the study.

\section{References}

1. Yamashita B, French $M$ (2011) Latent print development. In: Holder Jr EH Robinson LO, Laub JH (Eds), The fingerprint sourcebook. National Institute of Justice (NIJ), U.S. Department of Justice Office of Justice Programs, Washington, USA.

2. Gaensslen RE, Lee $H$, Harris $H(2007)$ Introduction to forensic science and criminalistics ( $1^{\text {st }}$ edn.), McGraw-Hill Higher Education, New York, USA.

3. Sarborough S, Henning R, Dechman G (2006) Advanced and innovative workflow for an AFIS configuration. J Forensic Identif 56: 526-533.

4. Federal Bureau of Investigation (2014) Next generation identification (NGI) 
Citation: Wang JZ. Quantitative Comparison of Partial-Full Fingerprints at Scenes Using a Hand-Held Digital Device: A Quasi-Experimental Design Study. J Forensic Investigation. 2016; 4(2): 6.

ISSN: 2330-0396

5. Komarinski P (2004) Automated fingerprint identification systems (AFIS) $\left(1^{\text {st }}\right.$ edn.), Elsevier, New York, USA.

6. Lipowicz A (2011) FBI deploys faster fingerprint ID system. Government Computer News (GCN)

7. Tabassi E, Wilson CL, Watson Cl (2004) Fingerprint image quality. Nationa Institute of Standards and Technology Interagency Report 7151. Nationa Institute of Standards and Technology, Gaithersburg, USA.

8. Kingston CR, Kirk PL (1965) Historical development and evaluation of the "12 point rule" in fingerprint identification. International Criminal Police Review.

9. (2010) AFIS technical requirements specification version 1 by Hillsborough County Sheriff's Office.

10. National Research Council (2009) Strengthening forensic science in the United States: a path forward. The National Academies Press, Washington DC, USA.
11. Busey T, Yu C (2014) The information content of friction ridge impressions as revealed by human experts. National Institute of Justice (NIJ), U.S Department of Justice, Washington, DC, USA

12. Ratha NK, Pandit VD, Bolle RM, Vaish V (2000) Robust fingerprint authentication using local structure similarity. Workshop on applications of computer vision, pp. 29-34

13. Jea TY, Govindaraju V (2005) A minutia-based partial fingerprint recognition system. Pattern Recognit 38: 1672-1684.

14. Jiang X, Yau WY (2000) Fingerprint minutiae matching based on the local and global structures. International Conference on Pattern Recognition Proceedings, pp. 1038-1041.

15. Yen R, Guzman J (2007) Fingerprint image quality measurement algorithm. J Forensic Identif 57: 274-287.

16. Schmitz A (2012) Psychology research methods: core skills and concepts. 